\title{
Neuromuscular Ultrasound in Amyotrophic Lateral Sclerosis
}

Jung Im Seok

Department of Neurology, School of Medicine, Catholic University of Daegu, Daegu, Korea

Ultrasound is a painless and one of the least invasive methods of medical diagnostic testing, which enables visualization of the anatomy of nerves as well as surrounding structures. Over the past decades, researchers have investigated the ultrasonographic changes that occur in the nerves and muscles of patients with motor neuron disease. The current article reviews the sonographic findings that are helpful in the diagnosis of amyotrophic lateral sclerosis. The utility of ultrasound in the assessment of respiratory function has also been described.

J Neurosonol Neuroimag 2019;11(1):73-77

Key Words: Ultrasonography; Amyotrophic lateral sclerosis; Diagnosis; Respiration

Received: April 16, 2019

Revised: April 29, 2019

Accepted: April 30, 2019

Address for correspondence: Jung Im Seok

Department of Neurology, School of Medicine, Catholic University of Daegu, 33 Duryugongwon-ro 17-gil, Nam-gu, Daegu 42472, Korea

Tel: +82-53-650-3440

Fax: $+82-53-654-9786$ E-mail:ji-helpgod@hanmail. net

\section{INTRODUCTION}

Electrodiagnosis (EDX) has traditionally been used as the method of choice for evaluation of peripheral nerves. However, EDX has two key limitations, namely the inability to provide anatomic details and patient discomfort. While magnetic resonance imaging (MRI) offers anatomic information, it is neither portable nor cost effective. Ultrasound is a painless and one of the least invasive methods of medical diagnostic testing, which enables visualization of the anatomy of nerves as well as surrounding structures. In the past decade, neuromuscular ultrasound has been used routinely in the assessment of entrapment neuropathies. Recently, the technique has been studied in a variety of neuromuscular disorders such as demyelinating polyneuropathy, leprosy, traumatic neuropathy, and motor neuron disease. The current review focuses on the use of neuromuscular ultrasound in amyotrophic lateral sclerosis (ALS).

\section{DIAGNOSIS OF AMYOTROPHIC LATERAL SCLE- ROSIS}

ALS is a neurodegenerative disease that affects both upper and lower motor neurons. The disease course is characterized by progressive weakness and muscle atrophy, leading to disability and eventually death. The diagnosis of ALS is challenging, because of the absence of a diagnostic marker and variability in presentation. Although EDX remains the method of choice in confirmation of the diagnosis of ALS, ultrasound may also be used for various purposes in cases of ALS, such as for nerve cross-sectional area (CSA) measurement, identification of fasciculation, and assessment of the split hand index.

Reduction in the cross-sectional area of motor nerves may occur in ALS. Cartwright et al. ${ }^{1}$ recently presented results of sonography evaluation, showing smaller CSA of the median nerve in the mid-arm of patients with ALS compared to the controls. Evaluation of cervical nerve roots might be more appropriate to detect loss of motor axons than peripheral nerves because the thinning of cervical roots is more pronounced in ALS. ${ }^{2-4}$

The El Escorial criteria have been used for decades for the diagnosis of ALS. ${ }^{5}$ Recent study has shown that the Awaji criteria are more sensitive than the revised El Escorial criteria for the diagnosis of ALS. ${ }^{6}$ One of the significant differences between the revised El Escorial and Awaji criteria is that in the latter, fasciculation 
was reintroduced as an evidence of acute denervation. Ultrasound is a particularly sensitive tool for detecting fasciculation in muscles, owing to the ability to assess deeper muscle layers, which is beyond the capacity of visual inspection or electromyography (EMG) needle investigation. The detection of fasciculation in the tongue is particularly valuable, given the importance of bilateral tongue fasciculation in the diagnosis of ALS. ${ }^{7}$ By applying the Awaji criteria, the proportion of patients in the definite category rose from 31\% with EMG to $53 \%$ with both EMG and ultrasound (US). ${ }^{8}$

In ALS, hand muscle wasting preferentially affects the 'thenar (lateral) hand', including the abductor pollicis brevis (APB) and first dorsal interosseous (FDI) muscles, with relative sparing of the hypothenar muscles (the abductor digiti minimi $[\mathrm{ADM}])$. The $\mathrm{APB} / \mathrm{ADM}$ ratio and FDI/ADM ratio, calculated simply by measuring the amplitudes of the compound muscle action potentials, are lower in patients with ALS than controls. This peculiar pattern of dissociated atrophy of the intrinsic hand muscles is termed the 'split hand' and is rarely seen in diseases other than ALS. ${ }^{9}$ Ultrasound can be used to assess the split hand index, using echointensity ratios of the thenar and hypothenar muscles. Ultrasound has been shown to be more sensitive in evaluating dissociated small hand muscle atrophy compared to EDX and may be a reliable diagnostic marker for differentiating ALS from other neuromuscular disorders and healthy controls. $^{10}$

\section{DIFFERENTIAL DIAGNOSIS FROM MULTIFO- CAL MOTOR NEUROPATHY}

As multifocal motor neuropathy (MMN) is a treatable disorder, differentiation of the condition from lower motor neuron disease is important. Diagnosis of MMN is based on high titers of anti-ganglioside $\mathrm{M}_{1}$ immunoglobulin M (anti-GMı IgM) antibodies and presence of multifocal conduction blocks outside of common nerve entrapment sites, without signs of upper motor neuron involvement. ${ }^{11}$ However, some patients with atypical MMN have no detectable conduction block or anti-GMr antibody. ${ }^{12,13}$ In MMN, focal nerve enlargement has been previously demonstrated on ultrasound. Recent studies have reported that MMN appears to pre- dispose to pathological enlargement of CSA in the forearm (median and ulnar nerves) and the tibial nerve at the ankle. ${ }^{14} \mathrm{~A}$ previous study reported a case of $\mathrm{MMN},{ }^{15}$ wherein the ultrasound image demonstrated focal enlargement of the median nerve in the forearm and ulnar nerve in the elbow (Fig. 1). Median values of all peripheral sensorimotor nerves and the vagus nerve were significantly enlarged in MMN in comparison with ALS and healthy controls. ${ }^{16}$ The mean diameters of $\mathrm{C}_{5}$ and $\mathrm{C} 6$ roots in patients with MMN were significantly larger than those in ALS and controls. ${ }^{3}$ The presence of nerve enlargement may be a useful discriminating factor between MMN and ALS.

\section{ASSESSMENT OF RESPIRATORY FUNCTION}

Respiratory muscle weakness is significant in ALS and respiratory failure is a common cause of death in this group of patients. Although several diagnostic tests are available for evaluation of the diaphragm, these are associated with limitations. ${ }^{17}$ Chest X-rays are relatively insensitive. Fluoroscopy and computed tomography involve radiation exposure and cannot be performed at the patient's bedside. MRI has the disadvantage of limited availability and high cost. Electromyography is uncomfortable and harbors the risk of pneumothorax. Pulmonary function test can be an unreliable measure of respiratory function in ALS patients with bulbar involvement, because orofacial weakness in ALS may cause air leakage around the mouthpiece. Ultrasound is a noninvasive technique that has proved to be an accurate, safe, easy-to-use, bedside modality that overcomes several limitations of other diagnostic techniques for evaluation of the diaphragm.

Two-dimensional B-mode ultrasound can be used to measure diaphragm thickness. Ultrasound scans of the bilateral diaphragm were obtained while the participant was in a supine position. To obtain an intercostal view in the zone of apposition, a transducer was placed at the anterior axillary line between the seventh and eighth or eighth and ninth ribs. In this view, real-time movement of the diaphragm was recorded on B mode and the diaphragm was outlined by the two hyperechoic lines of the pleural and peritoneal membranes (Fig. 2). The thickness of the diaphragm was measured by plac- 

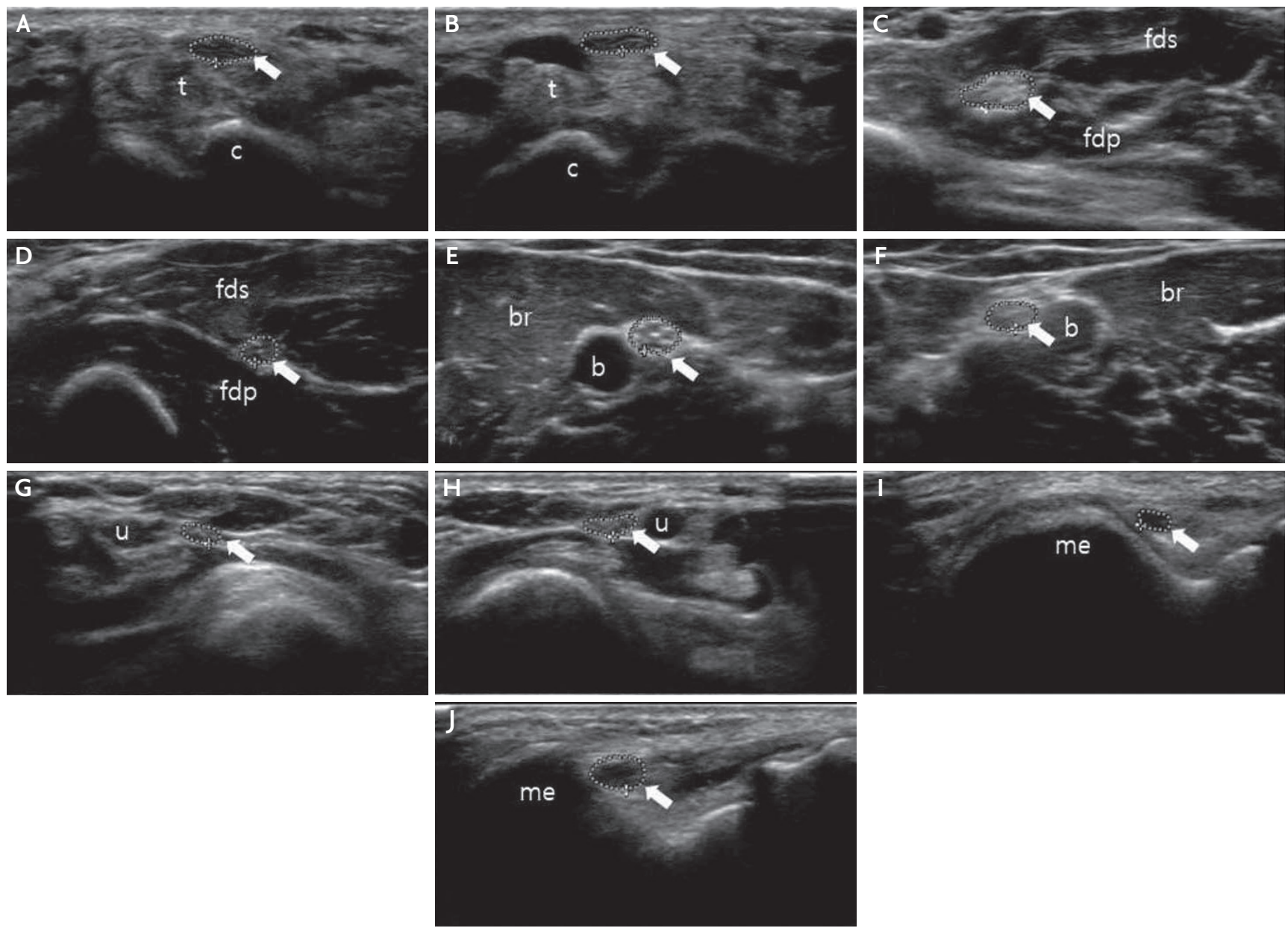

FIG. 1. Peripheral nerve (arrow) ultrasound in multifocal motor neuropathy. (A, B) The CSA of right (A) and left (B) median nerve at the wrist is normal. (C, D) The CSA of right (C) median nerve at the forearm increased to $15.2 \mathrm{~mm}^{2}$ and left (D) median nerve is normal $\left(5.9 \mathrm{~mm}^{2}\right)$. (E, F) The CSA of right $(E)$ and left $(F)$ median nerve at the upper arm is normal. $(G, H)$ The CSA of right $(G)$ and left $(H)$ ulnar nerve at the wrist is normal. (I, J) The CSA of right (I) ulnar nerve at the elbow is normal $\left(3.5 \mathrm{~mm}^{2}\right)$, but left $(J)$ ulnar nerve increased to $11.1 \mathrm{~mm}^{2}$. t; tendon, c; carpal bone, fds; flexor digitorum superficialis, fdp; flexor digitorum profundus, b; brachial artery, br; brachialis, u; ulnar artery, me; medial epicondyle, CSA; cross-sectional area.
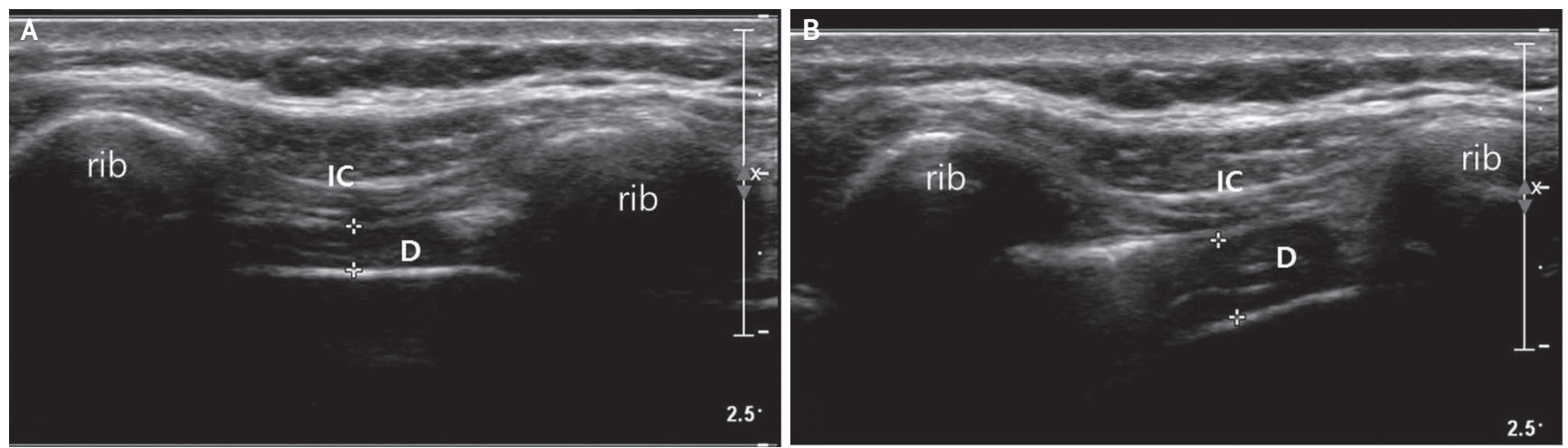

FIG. 2. Measurement of thickness of the diaphragm. (A) At the end of quiet expiration, the diaphragm is seen as a hypoechoic structure between two hyperechoic fascia. (B) At maximal inspiration, the diaphragm is seen 'peeling away' from the chest wall. The distance between two markings (+) means muscle thickness. The diaphragm thickness fraction was calculated as percentage using the following formula: (thickness at maximal inspiration - thickness at resting end expiration) / thickness at resting end expiration. IC; intercostal muscle, D; diaphragm. 
ing electronic calipers just inside the two hyperechoic lines where the lines were parallel (usually at the midline between two ribs). An average of the two images was considered for each measurement to obtain thickness values at the resting end expiration, resting end inspiration, and maximal inspiration phases. Thickness at maximal inspiration was measured at the point of maximal inspiration or at the point at which the diaphragm became obscured by the lung. Subsequently, the diaphragm thickness fraction (DTF) was calculated as percentage using the following formula: (thickness at maximal inspiration - thickness at resting end expiration) / thickness at resting end expiration.

Normal reference values for the thickness of the diaphragm and the DTF in a Korean population were reported. ${ }^{18}$ Although the DTF was affected less by demographic factors, the thickness of the diaphragm was significantly greater in men compared with women and was significantly correlated with weight, height, and body mass index (BMI). ${ }^{18}$ For identification of acute onset diaphragm paralysis, the DTF has been proposed to be more sensitive than measurement of thickness. In case of chronically paralyzed diaphragm, diaphragm is thin and does not thicken during inspiration. ${ }^{19}$ In such cases, therefore, both parameters including thickness and thickening ratio are useful. However, the thickness alone can be normal in cases of acute paralysis of the diaphragm.

In ALS patients, ultrasound of the diaphragm shows bilateral atrophy with minimal thickening during inspiration. Thickness of the diaphragm significantly correlated with the amplitude of the motor response by electric phrenic nerve stimulation and correlated to maximal voluntary ventilation and forced vital capacity. ${ }^{20}$ Monitoring for the development of respiratory muscle weakness in ALS is essential to evaluate the timing for non-invasive mechanical ventilation (NIV), because NIV increase survival time and improve quality of life. $\mathrm{PaCO}_{2}$ is a useful test to evaluate respiratory function in patients with ALS. The DTF was inversely correlated with $\mathrm{PaCO}_{2}$ and a DTF <39\% might be considered a warning of significant hypercapnia $\left(\mathrm{PaCO}_{2}\right.$ $>45 \mathrm{mmHg}$ ) in patients with ALS. ${ }^{21}$

\section{CONCLUSION}

In ALS, a combination of measurement of nerve thickness with recording of fasciculation and muscle echogenicity may improve the diagnostic potential of ultrasound. In addition, ultrasound can be used as a tool to assess respiratory function in patients with ALS.

\section{Conflicts of Interest}

No potential conflict of interest relevant to this article was reported.

\section{REFERENCES}

1. Cartwright MS, Walker FO, Griffin LP, Caress JB. Peripheral nerve and muscle ultrasound in amyotrophic lateral sclerosis. Muscle Nerve. 2011;44:346-351.

2. Nodera H, Takamatsu N, Shimatani Y, Mori A, Sato K, Oda $\mathrm{M}$, et al. Thinning of cervical nerve roots and peripheral nerves in ALS as measured by sonography. Clin Neurophysiol. 2014;125:1906-1911.

3. Nodera H, Izumi Y, Takamatsu N, Kaji R. Cervical root sonography to differentiate multifocal motor neuropathy from ALS. J Med Invest. 2016;63:104-107.

4. Mori A, Nodera H, Takamatsu N, Maruyama-Saladini K, Osaki Y, Shimatani Y, et al. Sonographic evaluation of cervical nerve roots in ALS and its clinical subtypes. J Med Invest. 2016;63:54-57.

5. Brooks BR, Miller RG, Swash M, Munsat TL; World Federation of Neurology Research Group on Motor Neuron Diseases. El Escorial revisited: revised criteria for the diagnosis of amyotrophic lateral sclerosis. Amyotroph Lateral Scler Other Motor Neuron Disord. 2000;1:293-299.

6. Carvalho MD, Swash M. Awaji diagnostic algorithm increases sensitivity of El Escorial criteria for ALS diagnosis. Amyotroph Lateral Scler. 2009;10:53-57.

7. Li TM, Day SJ, Alberman E, Swash M. Differential diagnosis of motoneurone disease from other neurological conditions. Lancet. 1986;2:731-733.

8. Misawa S, Noto Y, Shibuya K, Isose S, Sekiguchi Y, Nasu S, et al. Ultrasonographic detection of fasciculations markedly increases diagnostic sensitivity of ALS. Neurology. 2011;77:1532-1537.

9. Eisen A, Kuwabara S. The split hand syndrome in amyotrophic lateral sclerosis. J Neurol Neurosurg Psychiatry. 
2012;83:399-403.

10. Seok HY, Park J, Kim YH, Oh KW, Kim SH, Kim BJ. Split hand muscle echo intensity index as a reliable imaging marker for differential diagnosis of amyotrophic lateral sclerosis. J Neurol Neurosurg Psychiatry. 2018;89:943-948.

11. Olney RK, Lewis RA, Putnam TD, Campellone JV Jr; American Association of Electrodiagnostic Medicine. Consensus criteria for the diagnosis of multifocal motor neuropathy. Muscle Nerve. 2003;27:117-121.

12. Pakiam AS, Parry GJ. Multifocal motor neuropathy without overt conduction block. Muscle Nerve. 1998;21:243-245.

13. Van Asseldonk JT, Franssen H, Van den Berg-Vos RM, Wokke JH, Van den Berg LH. Multifocal motor neuropathy. Lancet Neurol. 2005;4:309-319.

14. Kerasnoudis A, Pitarokoili K, Behrendt V, Gold R, Yoon MS. Multifocal motor neuropathy: correlation of nerve ultrasound, electrophysiological, and clinical findings. J Peripher Nerv Syst. 2014;19:165-174.

15. Rha HJ, Seok JI, Lee SR. Multifocal motor neuropathy: complementary role of ultrasound. J Korean Neurol Assoc. 2018;36:119-121.
16. Grimm A, Décard BF, Athanasopoulou I, Schweikert K, Sinnreich M, Axer H. Nerve ultrasound for differentiation between amyotrophic lateral sclerosis and multifocal motor neuropathy. J Neurol. 2015;262:870-880.

17. Sarwal A, Walker FO, Cartwright MS. Neuromuscular ultrasound for evaluation of the diaphragm. Muscle Nerve. 2013;47:319-329.

18. Seok JI, Kim SY, Walker FO, Kwak SG, Kwon DH. Ultrasonographic findings of the normal diaphragm: thickness and contractility. Ann Clin Neurophysiol. 2017;19:131-135.

19. Seok JI, Kim KC, Rha HJ, Lee SR. Sonographic evaluation of the diaphragm in patients with unilateral diaphragmatic paralysis. Ann Clin Neurophysiol. 2018;20:93-96.

20. Fantini R, Mandrioli J, Zona S, Antenora F, Iattoni A, Monelli $\mathrm{M}$, et al. Ultrasound assessment of diaphragmatic function in patients with amyotrophic lateral sclerosis. Respirology. 2016;21:932-938.

21. Hiwatani Y, Sakata M, Miwa H. Ultrasonography of the diaphragm in amyotrophic lateral sclerosis: clinical significance in assessment of respiratory functions. Amyotroph Lateral Scler Frontotemporal Degener. 2013;14:127-131. 